\title{
Electron Beam Polarimetry for EIC/eRHIC
}

\author{
W. Lorenzon
}

Randall Laboratory of Physics, University of Michigan, Ann Arbor, Michigan 48109-1120, USA

\begin{abstract}
Ideas are being presented about how to measure the electron beam polarization at the Electron Ion Collider (EIC) at RHIC with high precision. Based on lessons learned at the HERA storage ring it is discussed which design and physics processes might be most appropriate for EIC. This is a summary of the ideas and concepts discussed at the first workshop on EIC electron beam polarimetry at BNL in November 2002.
\end{abstract}

\section{INTRODUCTION}

The current design of the EIC project at RHIC foresees collisions of 5-10 GeV longitudinally polarized electrons on $25-250 \mathrm{GeV}$ protons or $100 \mathrm{GeV} / \mathrm{u}$ heavy ions (such as gold). It is anticipated that the electron beam polarization is about $70 \%$ and that it needs to be measured with high precision $(\approx 1 \%$ syst.). Unfortunately, a polarized electron bunch has no macroscopic properties that could be useful for measuring its polarization, as was pointed out by Ref. [1]. It is argued that a polarized electron bunch represent a very weak magnetic dipole which has a strength that is roughly seven orders of magnitude less than a piece of magnetized iron of comparable size. Therefore, one is inevitably lead to consider microscopic processes, i.e. spin-dependent scattering processes. The simplest such processes are the elastic processes which have three very useful properties: a) the cross sections for elastic scattering are usually large, b) elastic scattering processes have simple kinematical properties, and c) the physics or elastic electron (positron) scattering are quite well understood.

There are currently three different targets used to measure the polarization of electron (positron) beams: nuclei, electrons, and photons. Mott scattering, or $e^{-}-$nucleus scattering is mainly used at low energies $(100-300 \mathrm{keV})$ to measure the polarization of electrons from polarized sources. Møller (Bhabha) scattering, or $e^{-}\left(e^{+}\right)-$electron scattering is widely used for polarized beams in the $100 \mathrm{MeV}$ to many $\mathrm{GeV}$ energy range. Unfortunately, it is destructive to the beam and therefore not suitable for storage rings. In contrast, Compton scattering, or $e^{ \pm}-$photon scattering, which is suitable for energies above $1 \mathrm{GeV}$, and ideal for energies above $10 \mathrm{GeV}$, is not destructive to beams in storage rings and is therefore the only choice to date for high energy storage rings, with the exception of a new idea discussed in Ref. [2].

There are many polarimeters that are, or have been, in use at various laboratories. Here, we describe a polarimeter that has taught as several lessons for how to design a polarimeter for EIC. It is the Longitudinal Polarimeter at the HERA accelerator at DESY [3]. 


\section{THE LONGITUDINAL POLARIMETER AT HERA}

At the HERA storage ring, the electron (positron) beam can become transversely polarized through the emission of synchrotron radiation (Sokolov-Ternov effect) with average asymptotic polarization values of $55 \%$ and typical rise times of 22 minutes. The Longitudinal Polarimeter is the second polarization monitor at HERA that is based on Compton back-scattering of circularly polarized photons from an laser beam [3]. In contrast to the Transverse Polarimeter, which measures the transverse polarization of the electron beam in the HERA West section [4], it provides a measurement of the longitudinal beam polarization in the East section of HERA between the spin rotators at the HERMES experiment, as shown in Fig. 1.

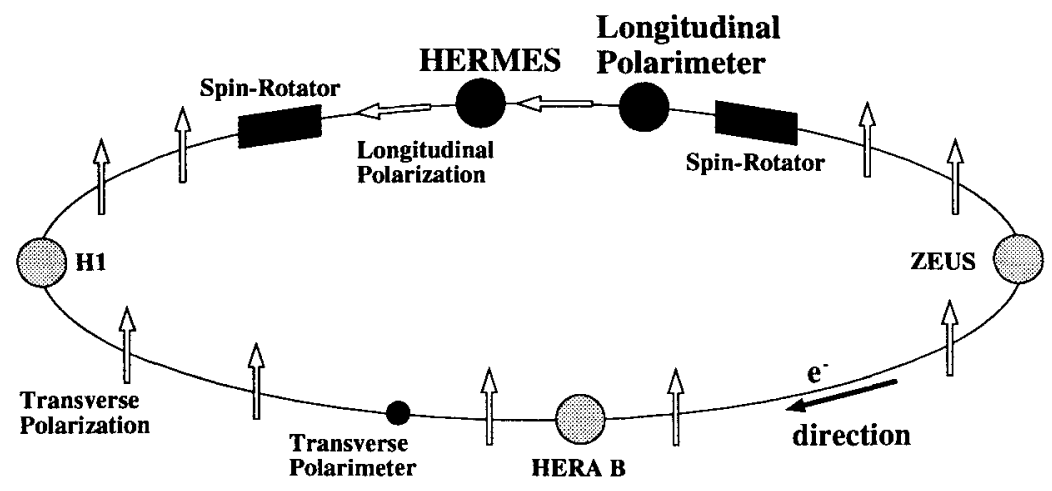

FIGURE 1. The electron ring layout at HERA.

The Longitudinal Polarimeter consists of two main components, a laser beam and a calorimeter that measures the back-scattered Compton photons. The longitudinal polarization can be obtained from the measurement of the energy-dependent asymmetry under reversal of the laser photon helicity using either the single-photon or the multiphoton mode. However, due to intense bremsstrahlung background from the HERMES target and the long straight section, the multi-photon mode is used [3]. In contrast to the single-photon mode, in which the energy of each individual Compton photon is analyzed, in the multi-photon mode one measures the total energy deposited in the detector by many Compton photons per laser pulse interaction with an electron bunch.

The advantages of running in single-photon mode would be twofold. The asymmetries are large, up to $60 \%$ for the conditions at HERA (532 nm laser light scattered off 27.5 $\mathrm{GeV}$ electrons), and the energy spectra can be compared to the Compton cross sections. Operating the Longitudinal Polarimeter in multi-photon mode has the advantage of being effectively independent of bremsstrahlung background in the HERA storage ring.

The longitudinal polarization of each electron bunch is determined by switching between the two light helicity states and analyzing the two resulting energy distributions. This calculation is provided every minute. The average polarization of the electron beam is finally computed as the mean of the individual bunch polarizations weighted by the corresponding time-averaged bunch currents. The Longitudinal Polarimeter can also measure the polarization of individual bunches with an absolute statistical accuracy of $3 \%$ in twenty minute long measurements. 


\section{POLARIMETRY AT EIC}

Experience at the HERA storage ring has demonstrated that it is imperative to include polarization diagnostics and monitoring capabilities in the design of the electron beam lattice. The specifics depend on the design of the electron machine, and are much more crucial for a ring option than for a linac option. In either case, one has to ensure that the beam polarization can be measured continuously during data taking to minimize systematic uncertainties associated with the beam polarization. For a linac, destructive (Møller scattering with an iron foil) or non-destructive methods (Compton scattering or Møller scattering with an atomic beam source) can be employed.

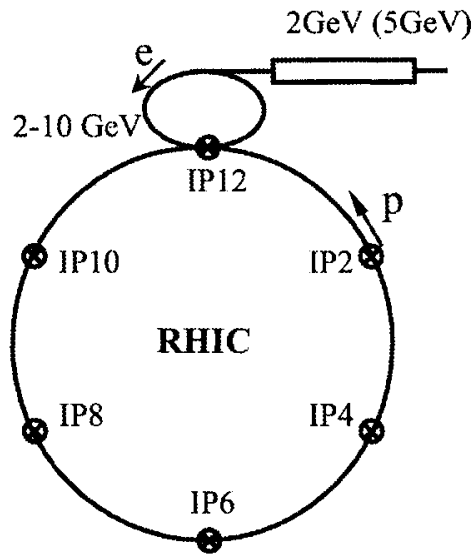

FIGURE 2. A possible layout for the EIC at Brookhaven National Laboratory.

The most recent design favors the ring option, as shown in Fig. 2. For a 5-10 GeV electron beam, there are essentially two options: either to measure the transverse polarization of the beam away from the target (as it is done at HERA with the Transverse Polarimeter), or to measure the longitudinal polarization close to the target (as it is done at HERA with the Longitudinal Polarimeter). The longitudinal polarization is measured via rate or energy asymmetries, which are generally much easier to measure than spatial asymmetries as in the case of transverse polarization. Nevertheless, having two independent measurements with vastly different systematic uncertainties should be strongly considered, if a precision of $1 \%$ (or better) needs to be achieved. If only one polarimeter is considered, it should be a longitudinal type polarimeter.

There are three components to be considered. The laser or laser transport system, the laser-electron interaction region, and the Compton detector.

Maybe the most important component is the location of the laser-electron interaction region. Its location has to be chosen to minimize bremsstrahlung and synchrotron radiation, and to optimize the rate of the back-scattered Compton photons versus the background rate. Minimizing bremsstrahlung background requires to have a short section of beam line, like introducing a chicane with soft bends to also minimize synchrotron background. This was done for the Transverse Polarimeter at HERA, and resulted in very small background rates, while for the Longitudinal Polarimeter, this was not possible (changing the lattice after it was built was too expensive) and resulted in much larger 
background rates. Maximizing the Compton rate means that the crossing angle between the laser beam and the electron beam should be as small as possible (of order 3-10 mrad), and the horizontal widths of the electron and laser beams should both be small $(0.5 \mathrm{~mm}$ or smaller). In addition, the transverse spatial distribution of the back-scattered Compton photons due to the size and divergence of the electron beam has to be kept small if a) the Compton detector is far away (10s of meters), or b) if the transverse polarization needs to be measured, otherwise it will lead to a dilution of the spatial asymmetry.

One has to consider whether to build an optical cavity (which is expensive and delicate, and cannot be accessed during beam operation) or whether to build a more conventional laser (which can be accessed at all times) plus laser transport system. If rates, and therefore statistical precision allow it, a conventional laser transport system is more conservative. At low energies $(<10 \mathrm{GeV})$ it is advantageous to use optical or maybe even UV laser light (as compared to IR light) because the analyzing power is proportional to the product of the electron and the photon energy. In addition, the energy of the Compton edge (which corresponds to the maximum energy of the back-scattered Compton photons) is proportional to the photon energy. The higher the Compton edge energy, the easier it is to detect it and to distinguish the Compton photons from background.

The Compton detector should be radiation hard and fast $(<35 \mathrm{~ns})$ to be able to record signals for each individual bunch crossing. Calorimeters based on detecting Čerenkov light might be better suited than those based on detecting scintillation light, because they are inherently faster. The Compton detector should also be able to determine the impact position of the Compton photons. That could be achieved with the segmentation of the the detector or with an external position sensitive device.

Every effort should be made to keep the background conditions low. This allows to operate the Compton polarimeter in single-photon mode and to monitor the linearity of the detector, because it provides three energy calibration points, one at the beam energy (bremsstrahlung edge), one the Compton edge, and one at the zero crossing of the asymmetry (corresponding to $90^{\circ}$ scattering in the electron rest frame).

\section{ACKNOWLEDGMENTS}

I wish to thank Abhay Deshpande, Bill Franklin, Eugene Chudakov and Peter Schüler for many fruitful discussions. The author's research is supported in part by the U.S. National Science Foundation, Intermediate Energy Nuclear Science Division under grant No. PHY-0072297 and PHY-0244842.

\section{REFERENCES}

1. Schwartz, M. L., Physics with polarized electron beams, Tech. Rep. SLAC-PUB-4656, Stanford Linear Accelerator Center, Stanford University, Stanford, CA 94309 (1988).

2. Chdukov, E. A., and Luppov, V. G., Møller polarimetry with atomic hydrogen tagets, Tech. rep., Ann Arbor, MI 48109 (2002).

3. Beckmann, M. et al., Nucl. Instr. Meth. A, 479, 334 (2002).

4. Barber, D.P. et al., Nucl. Instr. Meth. A, 329, 79 (1993). 